4. H. E. Vaughan, On locally compact, metrisable spaces, Bull. Amer. Math. Soc. vol. 43 (1937) pp. 532-535.

5. G. T. Whyburn, A certain transformation on metric spaces, Amer. J. Math. vol. 54 (1932) pp. 367-376.

University of Southern California ANd UNIVERSITY OF WASHINGTON

\title{
A THEOREM OF ELIE CARTAN
}

G. A. HUNT

André Weil [1] and Hopf and Samelson [2] have given a topological proof of the following theorem of Elie Cartan.

Two maximal Abelian subgroups of a compact connected Lie group $G$ are conjugate within $G$.

I present a simple metric proof.

Lemma. If $x$ and $y$ are elements of the Lie algebra $g$ of $G$ then $\left[x, A_{\sigma} y\right]$ vanishes for some inner automorphism $A_{\sigma}$ of $G$.

Proof. Because $G$ is compact one can define on $g$ a nonsingular bilinear form $(u, v)$ which is invariant: $([u, v], w)+(v,[u, w]) \equiv 0$. We choose $\epsilon$ in $G$ so that $\left(x, A_{\sigma} y\right)$ attains its minimum for $\sigma=\epsilon$; without loss of generality we may assume $\epsilon$ to be the neutral element of $G$, and then $A_{\mathfrak{c}} y=y$. If now $z$ is any element of $g$ the function $\left(x, A_{\exp (t z)} y\right)$ has a minimum for $t=0$, so that its derivative vanishes there. Thus, keeping in mind that

$$
\left.\frac{d}{d t} A_{\exp (t z)} y\right|_{t=0}=[z, y],
$$

we have $(x,[z, y])=0$. From this equation and from the invariance of the bilinear form it follows that $([x, y], z)=0$ for all $z$; this can happen only if $[x, y]$ vanishes, for the bilinear form is nondegenerate.

Before proving Cartan's theorem I recall some well-known facts: A maximal Abelian subgroup $\mathfrak{H C}$ of $G$ is a torus group; there is an element $x$ in the Lie algebra $\mathfrak{h}$ of $\mathcal{H C}$ such that the one parameter group $\exp t x$ is dense in $\mathfrak{H C}$; if $y$ belongs to $g$ and $[x, y]=0$, then $y$ must lie in $\mathfrak{h}$.

Matters being so, let $\mathcal{J C}^{\prime}$ be a second maximal Abelian subgroup of $G$ and $x^{\prime}$ an element of its Lie algebra bearing the same relation

Received by the editors May 20, 1955. 
to $\mathcal{F C}^{\prime}$ as $x$ does to $\mathcal{H C}$. Now choose $\sigma$ in $G$ so that $\left[x, A_{\sigma} x^{\prime}\right]$ vanishes. Then $A_{\sigma} x^{\prime}$ lies in $\mathfrak{h}$; consequently $A_{\sigma}\left(\exp t x^{\prime}\right) \equiv \exp \left(t A_{\sigma} x^{\prime}\right)$ lies in $\mathfrak{H C}$ for every $t$. So $\mathfrak{H C}$, being closed, includes the closure $A_{\sigma}\left(\mathcal{F C}^{\prime}\right)$ of the oneparameter group $A_{\sigma}\left(\exp t x^{\prime}\right)$. Finally $A_{\sigma}\left(\mathcal{F C}^{\prime}\right)=\mathcal{H}$, because both are maximal Abelian subgroups of $G$.

Since every element of $G$ can be written as exp $y$, the argument shows that every element of $G$ can be moved into $\mathcal{H C}$ by an inner automorphism of $G$.

The referee has pointed out that the argument of the lemma above is very like one used by R. Bott [3] in another context.

\section{BIBLIOGRAPHY}

1. A. Weil, Démonstration topologique d'un théorème fondamental de Cartan, C. R. Acad. Sci. Paris vol. 200 (1935) pp. 518-520.

2. H. Hopf and H. Samelson, Ein Satz über die Wirkungsräume geschlossene Liescher Gruppen, Comment. Math. Helv. vol. 13 (1941) pp. 240-251.

3. R. Bott, On torsion in Lie groups, Proc. Nat. Acad. Sci. U.S.A. vol. 40 (1954) pp. 586-588.

Cornell University 